\title{
1 Characterization of suboxic groundwater colloids using a multi-method approach
}

2 Dan J. Lapworth ${ }^{\dagger, *}$, Björn Stolpe ${ }^{\ddagger}$, Peter J. Williams ${ }^{\dagger}$, Daren C. Gooddy ${ }^{\dagger}$, Jamie R. Lead ${ }^{\ddagger, \S}$

3 British Geological Survey, Maclean Building, Wallingford, Oxfordshire, OX10, 8BB, United

\section{$4 \quad$ Kingdom}

5 School of Geography, Earth and Environmental Sciences, University of Birmingham,

6 Edgbaston, Birmingham, B15 2TT, United Kingdom

7 Arnold School of Public Health, University of South Carolina, Columbia, South Carolina,

8 USA

$9 \quad{ }^{\dagger}$ British Geological Survey

$10 \ddagger$ University of Birmingham

$11{ }^{\S}$ University of South Carolina

12 * Corresponding author phone: (+44) (0)1491 692327; e-mail: djla@bgs.ac.uk 


\section{Abstract}

2

3 Anoxic groundwater colloid properties were measured using a minimally perturbing procedure for

4 sampling, processing and analysis. Analytical methods included atomic force microscopy (AFM),

5 flow field flow fractionation (FIFFF), transmission and scanning electron microscopy (TEM and

6 SEM). Shallow groundwater samples showed abundant iron rich nanoparticles (NP) with diameters of

$7 \quad 10-30 \mathrm{~nm}$ as well as a smaller heterogeneous polydisperse dissolved organic matter (DOM) fraction.

8 AFM results showed NP with average heights of $10 \pm 2 \mathrm{~nm}$, which was corroborated by high

9 resolution TEM and SEM. FIFFF with UV254 nm detection found particles with number average

10 diffusion coefficients of $2-3 \times 10^{-10} \mathrm{~m}^{2} \mathrm{~s}^{-1}$ and hydrodynamic diameters between $1.5-2 \mathrm{~nm}$, probably

11 representing smaller organic macromolecules. Aeration of the samples resulted in extensive

12 agglomeration of NP to form larger $(>50 \mathrm{~nm})$ colloids, and a reduction of UV-absorbing material in

13 the 0.5-4 $\mathrm{nm}$ range. The complementary methods described have potential applications for

14 investigating the fate and transport of NP in suboxic hotspots such as leachate plumes, waste water treatment plants and within the hyporheic mixing zone. 
1 Abstract Art

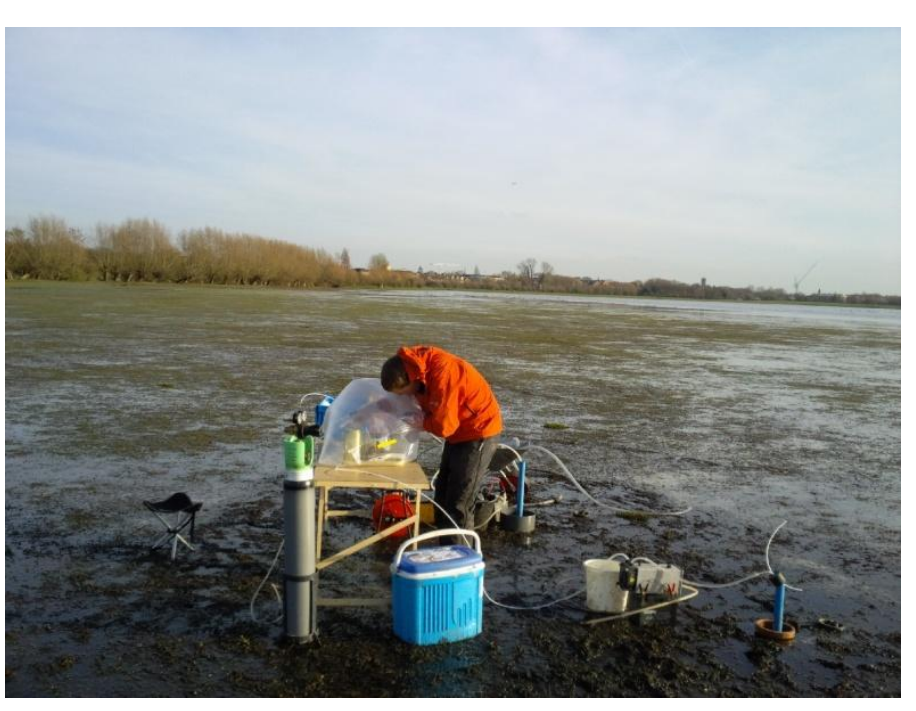

3 


\section{Introduction}

3 Natural nanoparticles (NP), particles with one dimension between 1-100 nm, and colloidal material,

4 particles with one dimension between $1 \mathrm{~nm}$ and $1 \mu \mathrm{m}$, are important vectors for contaminants in the

5 environment, and include iron or silica mineral particles, complex organic molecules such as humic-

6 like substances, or material derived from bacterial sources. ${ }^{[1-3]}$ Particles spanning these dimensions

7 can often have a dominant role in controlling speciation and enhancing the mobility of contaminants

8 in aquatic environments. ${ }^{[3]}$ They can also attenuate contaminant transport in some cases, and are

9 known to have an important role in biogeochemical cycling, and bioavailability of toxic substances. ${ }^{[4,}$

11 Many studies have used operationally-defined techniques such as cross-flow-filtration to investigate element association to colloidal material in environmental samples ${ }^{[6-8]}$ including in suboxic environments. ${ }^{[9]}$ Recent research on characterising groundwater NP has also focussed on understanding radionuclide fate and transport ${ }^{[10,11]}$ due to the potential risk of pollution from longterm underground radioactive waste storage facilities. Quantifying the size, structure and surface chemistry of colloids is important for understanding pollutant-colloid interactions. ${ }^{[12-14]}$

Developments in analytical methods e.g. flow field flow fractionation (FIFFF), atomic force microscopy (AFM), scanning electron microscopy (SEM) and transmission electron microscopy (TEM), have enabled the detailed characterisation of natural colloidal material. ${ }^{[15-18]}$ There has been particular attention paid to the importance and role of small $(<20 \mathrm{~nm}) \mathrm{NP}$ in trace element occurrence due to their relatively high specific surface area. ${ }^{[19]}$

FlFFF has been successfully coupled with a range of detectors (e.g. fluorescence, UV-vis, light scattering, ${ }^{[20]} \mathrm{AFM},{ }^{[18]}$ and inductively coupled plasma-mass spectroscopy ${ }^{[21-23]}$ ), to characterise fractionated particles. Importantly, FlFFF and AFM can cover the same size-range, provide detailed structural information on size, shape, diffusion coefficient and softness, and work under ambient redox conditions. 
1 Characterising suboxic environmental NP requires careful protocols for both sampling and analysis to

2 preserve the native redox status. For example, some aquifers or waste water systems may be partly or

3 completely lacking dissolved oxygen (DO). Subsequent introduction of $\mathrm{O}_{2}$ can lead to the oxidation of

4 ferrous iron to form colloidal iron oxide. ${ }^{[24,25]}$ In addition, sampling at low flow rates (ca. $100 \mathrm{~mL}^{-1}$

$5 \mathrm{~min}$ ) following borehole purging is required to collect representative groundwater samples. ${ }^{[26]}$ Due to

6 the challenges of maintaining suboxic conditions, in both sampling and analysing NP, very few

7 studies to date have successfully used modern state-of-the-art techniques to characterise nano-scale

8 particles under environmental conditions. However, many NP contaminant hot spots e.g. parts of

9 sewerage treatment processes, landfill leachate plumes and the hyporheic zone, can be classified as

10 suboxic environments. There is therefore a clear need to develop robust methods to characterise and

11 investigate the physiochemical properties and fate of both natural and introduced NP within these

12 environments.

13 In this study we have developed and piloted a procedure for sampling and characterizing

14 physiochemical properties of nano-scale particles in natural suboxic waters using a range of 15 complimentary methods: FIFFF and AFM, TEM and SEM. The procedure was used to investigate organic and iron-rich nanoscale particles $(<30 \mathrm{~nm})$ within a shallow alluvial groundwater in the

17 Thames floodplain, United Kingdom, locally impacted by a landfill leachate plume. These techniques 18 also have potential wider applications for studying the occurrence and fate of natural and engineered 19 nano-scale particles within other suboxic pollution hotspots. 


\section{Experimental}

2 Sampling. The field site (Figure S1 supplementary information) is located down gradient of a 3 landfill site in the floodplain of the River Thames, Oxford, United Kingdom [51 ${ }^{\circ} 46^{\prime} 5.18^{\prime}$ ' N, $1^{\circ} 16^{\prime}$, 4 47.67'W]. A pilot sampling round in December 2010 was used to test the sampling protocol and assess the stability of the samples prior to characterisation by AFM. Once the sampling and storage procedure had been tested in the December round and evaluated using AFM, the suboxic sampling methodology was extended to other characterisation techniques. In subsequent rounds (February 2011) samples were sampled and analysed by AFM, SEM and TEM; in the April 2011 round samples were analysed by FlFFF.

At two piezometer nests (sites 26 and 28) groundwater from the floodplain Terrace Gravels was sampled from $1.5 \mathrm{~m}$ (piezometer c) and $3.5 \mathrm{~m}$ (piezometer d) below ground level. In the December round piezometric heads in the piezometers were all below ground level, in the February round the piezometric head in $28 \mathrm{c}$ and $28 \mathrm{~d}$ was above ground level by $10 \mathrm{~mm}$, and the area around this nest flooded. In April 2011 they were below ground level at both sites. NP suboxic sampling was carried out in a portable chamber with an inert atmosphere $\left(\mathrm{BOC}^{\mathrm{Tm}}\right.$ oxygen free $\mathrm{N}_{2}$ gas). Piezometers were purged (minimum of 3 borehole volumes) and sampled using a peristaltic pump at a flow rate of 100 $\mathrm{mL} /$ minute. DO concentrations in both the pumped groundwater and the chamber were monitored continuously prior and during sampling. Samples were stored in sealed high density polyethylene

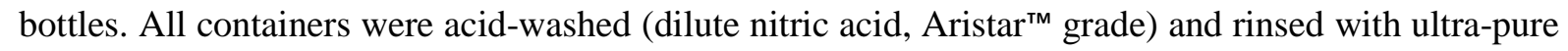
water $\left(\mathrm{R}>18.2 \mathrm{M} \Omega \mathrm{cm}^{-1}\right)$ prior to sampling. A surrounding jacket of airtight suboxic groundwater was used to protect the sample from DO diffusion prior to analysis, in a similar manner to methods used for groundwater dating using chlorofluorocarbons ${ }^{[27]}$ (See Figure 1). Samples were filtered using two $45 \mathrm{~mm}$ diameter in-line $0.45 \mu \mathrm{m}$ (Milipore $^{\mathrm{TM}}$ ) membrane filters in the field to remove larger particulate material, which is known to result in rapid aggregation. ${ }^{[28,29]}$ More detail on the sampling processs and groundwater chemistry are provided in Supplementary Information. 
1 Evaluation of Sampling and Storage Methodology. In suboxic waters, where dissolved Fe and Mn

2 are present in reduced Fe (II) and Mn (II) forms, contact with oxygen result in these species being

3

4 oxidised, via Fe(III) and Mn (III, IV), to form colloidal Fe and Mn oxide phases. ${ }^{[30]}$ This will potentially result in a shift in the distribution of Fe/Mn NP to larger sizes due to aggregation. To test the robustness of suboxic sampling and analytical procedure, an aliquot of sample was aerated by gently bubbling with compressed air for 10 minutes. This was carried out under laboratory conditions to investigate the evolution of NP during aerationand compare NP size distributions using AFM and FlFFF with aliquots that had been sampled using the suboxic methodology. As well as during sampling, DO was monitored in the samples before and after carrying out the preparation for AFM to ensure that samples were not contaminated with atmospheric oxygen during storage and transport.

Atomic Force Microscopy. AFM determines the height of NP, after sorption to a suitable flat substrate (mica in this case), by exploiting the repulsive and attractive forces between the sample and the silicon cantilever mounted instrument tip . All sample preparation and analysis with AFM were carried out within 36 hours of groundwater sampling to minimise any changes in NP composition during sample storage. The groundwaters (suboxic and aerated aliquots) were transferred to $5 \mathrm{~mL}$ vials under a nitrogen atmosphere. This method specifically focuses on: a) small diffusible NP and b) NP which strongly bind to the mica. The mica sheets were then rinsed by immersing in suboxic ultrapure water for a few seconds (to remove slats and loosely adhered materials) followed by drying under $60 \%$ humidity in nitrogen atmosphere for 30 minutes. With the AFM-instrument (Park System XE-100) placed in a laminar glove box with nitrogen-atmosphere, AFM-images were acquired over different areas (e.g. $0.5 \times 0.5 \mu \mathrm{m}, 2 \times 2 \mu \mathrm{m}, 20 \times 20 \mu \mathrm{m}$ ) of the mica, in non-contact mode with a $42 \mathrm{~N} \mathrm{~m}^{-}$ ${ }^{1}$ force constant and $330 \mathrm{kHz}$ frequency. For each sample, the NP height distribution of $>190$ particles was determined by measuring the maximum NP height above the mica of 25-35 NP on at least 6 different $2 \times 2 \mu \mathrm{m}$ images. The relationships $\Sigma n_{i} z_{i}^{2} / \Sigma n_{i} z_{i}$ and $\Sigma n_{i} z_{i} / \Sigma n_{i}$ were used to calculate the weight $S(z)$ and number $N(z)$ average particle heights respectively for each sample where $n_{i}$ is the number and $z_{i}$ is the height of each particle measured. The polydispersity $(P)$ of the samples was evaluated using the relationship $S(z) / N(z){ }^{[15]}$ 
1 Flow Field Flow Fractionation. FlFFF is a technique that determines the hydrodynamic diameter

2 distribution of NP based on their interaction with a cross flow field while they are eluted along a flow

3

4

5

6

7

8 channel. ${ }^{[15]}$ The asymmetrical FIFFF instrument (AF 2000, Postnova Analytics) had a channel defined by a $0.35 \mathrm{~mm}$ spacer and a $1 \mathrm{kDa}$ nominal cut-off ultrafiltration membrane of regenerated cellulose (Postonva Analytics). The suboxic FlFFF-carrier solution was made up of $10 \mathrm{mM} \mathrm{NaCl}$ at $\mathrm{pH} 8.5 \mathrm{~mL}$ of the groundwater samples (suboxic and aerated aliquots) were injected and focused in the channel for $30 \mathrm{~min}$ with a tip flow of $0.5 \mathrm{~mL} \mathrm{~min}{ }^{-1}$ and a focus flow of $2.5 \mathrm{~mL} \mathrm{~min}$, followed by elution and

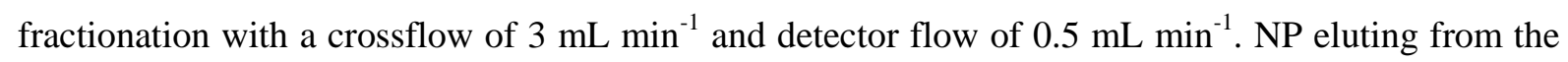
FlFFF-channel were detected on-line with UV-absorbance at 254, 350, 400, 575 and $700 \mathrm{~nm}$ and fluorescence at excitation-emission pair $350 / 450 \mathrm{~nm}$ which is specific to humic material. The continuous size distributions of NP were determined by converting retention time into diffusion coefficient. Equivalent hydrodynamic diameter distribution $\left(\mathrm{d}_{\mathrm{H}}\right)$ was calculated using the FlFFFtheory based on the Stokes-Einstein relationship, ${ }^{[31]}$ after calibrating the FIFFF channel thickness using two proteins (bovine serum albumin and ferritin) with known diffusion coefficients. Number average diffusion coefficients $D_{N}$ for particles in the $0-5 \mathrm{~nm}$ range were calculated using the relationship $\Sigma m_{i} D_{i}^{2} / \Sigma m_{i} D_{i}$ where $m$ is the mass based signal obtained by UV254 absorbance, proportional to concentration of organic matter, and $D$ is the diffusion coefficient calculated using FlFFF theory.

Transmission and Scanning Electron Microscopy. SEM and TEM are imaging techniques based on the electrons emitted/backscattered from (SEM) or transmitted through (TEM) the sample exposed to an electron beam. ${ }^{[32]}$ TEM and SEM are able to distinguish certain types of NP in complex samples, quantify their size and morphology, and have been used to calculate fractal dimensions of e.g. humic substances. ${ }^{[17]}$ In combinations with energy dispersive X-ray (EDX), measuring the energy of characteristic X-rays emitted from the sample under the electron beam, TEM and SEM can be used to determine the major element composition of individual NP. ${ }^{[1]}$ Samples for TEM and SEM were prepared under nitrogen atmosphere, by placing droplets of the samples (sub-oxic and aerated aliquots) on Formvar/carbon coated 300 mesh Cu TEM grids placed horizontally on a clean surface. 
1 After $30 \mathrm{~min}$, the grids were rinsed by immersing in suboxic ultrapure water for a few seconds,

2 followed by drying in a nitrogen atmosphere. TEM (JEOL 1200EX) images were acquired at $80 \mathrm{keV}$,

3 on five different areas of the grids using a range of magnifications (30 000-500 000).. SEM images

4 (JEOL 7000) with associated EDX spectra were acquired on three different particles for each sample

5 as well as on the background grids. All microscopy was carried out within two weeks of sampling.

6 Although the TEM grids were prepared shortly after sampling, and dried under nitrogen to minimise

7 oxidation, some grids were stored in air after drying for several days prior to analysis. The formation

8 of NP and colloids as a result iron and manganese oxidation of the dried samples is therefore possible

9 but minimal. 


\section{$1 \quad$ Results and Discussion}

2 Assessment of suboxic sampling protocol. Figure 2 shows examples of AFM scans $(1 \times 1 \mu \mathrm{m})$ of NP

3 from each site with and without aeration. The AFM NP size range for suboxic samples were found to

4 be between 1-20 nm, while those which were aerated had NP had mean heights of $>50 \mathrm{~nm}$, and in

5 some cases these reached sizes of $>100 \mathrm{~nm}$. DO concentrations in samples prior to preparation were $<0.5 \mathrm{mg} / \mathrm{L}$, and had not altered during storage. These results show the rapid formation and agglomeration of NP following contact with air, resulting in a dramatic shift in the NP size distribution. This demonstrates that maintaining suboxic conditions during storage and preparation is important. The shift in particle size and properties was also corroborated by FIFFF and SEM data and provides strong evidence that the NP distributions in aliquots that were sampled and processes under suboxic conditions are representative of true environmental conditions. Filtering the samples prior to NP characterisation is important to reduce aggregation, microbiological activity, and the potential breakdown of organic and mineral phases, as well as sorption/desorption reactions on particulate phases.

Nanoparticle size distributions. Absorbed NP height distributions detected by AFM for the suboxic samples from all four sites are graphically summarised as kernel-density estimates in Figure 3a-d. Median values for the samples in each case are shown as vertical lines in Figure 3. It is clear from these plots that none of the samples follow a normal distribution, as confirmed for all samples using a Shapiro-Wilks test ${ }^{[33]}$ for normality $(p<0.05)$. Results of a Kruskal-Wallis ${ }^{[34]}$ test were found to be significant $\left(p<2 \times 10^{-16}\right)$; the mean ranks of particle heights are significantly different for the 7 samples. Median values for all sites were close to $10 \mathrm{~nm}$ (see Figure 3). However, these comparisons do not represent the complex polymodal distribution found in all samples. The proportion of adsorbed particles observed by AFM that were in the 0-5 $\mathrm{nm}$ size range, typical sizes for humic-like macromolecules, varied between $5-50 \%$, and for most samples was only found to be approximately $10 \%$. However, the smaller particles may be over represented due to the relatively short adsorption time (30 min) used in the sample preparation. Sites from the more shallow locations (26c and $28 \mathrm{c}$ ) had bi-modal distributions, and were less skewed compared to the samples from the deeper sites. The 
three samples from the deeper sites had more complex distributions with a greater proportion of larger outliers with particle heights $>25 \mathrm{~nm}$. Using a Wilcoxon rank sum test ${ }^{[35]}$, samples from the two shallow sites $26 \mathrm{c}$ and $28 \mathrm{c}$ were found to have significantly different mean ranks of particle height for the two different sampling rounds $\left(p<6 \times 10^{-14}\right.$ ), while for sample $26 \mathrm{~d}$ this was not found to be significant $(p=0.79)$. This may suggest that the particle distribution in the shallow sites show comparatively greater temporal variability.

Average polydispersity index $(P)$ (Table 1 ) were found to be comparable for the two shallow groundwaters, ca.1.1, while they were higher for the two deeper sites (1.4 and 2.1 respectively). This suggests that there is an increase in NP heterogeneity with depth. This is likely due to a range of processes including microbiological processing of DOC in the leachate plume, redox and pore-water diffusion controlled processes as well as, seasonally dynamic recharge and mixing of waters. ${ }^{[36,37]}$ In addition, the groundwater chemistry (Table S1, supplementary information) shows that the deeper sites are also affected to a greater extent by the landfill leacheate plume (e.g. higher DOC, SEC, $\mathrm{HCO}_{3}$ ) which is likely to be a major factor driving the geochemical processes and colloid generation in this shallow aquifer.

For all sites there were two dominant populations, one with a median particle height of ca. $5 \mathrm{~nm}$ and a second with a particle height of ca. $15 \mathrm{~nm}$, with overlapping distributions. In the shallow sites these were found to change in their relative proportions during the two sampling rounds. For example, at site 26c (Figure 3a), the larger population dominated the December round, while the smaller population dominated the February round. The reverse was observed for site 28c (Figure 3c). These results suggests that the NP distributions in the deeper sites are less dynamic compared to those in the shallow samples which showed marked shifts in distributions between sampling rounds. Overall, these observed differences are likely to be due to mixing and recharge processes in the shallow groundwaters influenced in part by regular inundation within the alluvial floodplain. The pre (first round) and post (second round) inundation conditions at site $28 \mathrm{c}$ could perhaps explain the observed changes in DOM within the shallow aquifer at this site, with a shift to larger NP caused by particle agglomeration due mixing of suboxic groundwater with soil water and surface water as groundwater 
1 levels rise with the onset of inundation. One explanation for these different populations is that the

2 larger particles represent agglomerations of smaller particles. Alternatively, they are different types of

3 NP, perhaps the small NP representing humic/fulvic like organic matter while the larger NP represents

4 iron rich mineral NP. ${ }^{[15,38]}$ These possibilities were further explored by FlFFF coupled to UV and

5 fluorescence detection.

6 Hydrodynamic diameters of UV-absorbing material. Continuous $0.1-10 \mathrm{~nm}$ hydrodynamic 7 diameter and diffusion coefficient distributions of UV-absorbing material, determined by FlFFF, are 8 shown in Figure 4. Diffusion coefficients of suboxic samples were distributed between $1-5 \times 10^{-10}$ $9 \mathrm{~m}^{2} \mathrm{~s}^{-1}$, with maximum values between $2-4 \times 10^{-10} \mathrm{~m}^{2} \mathrm{~s}^{-1}$, see Figure $4 \mathrm{a}$. The hydrodynamic diameter of UV-absorbance at $254 \mathrm{~nm}$ (UV254) was mainly distributed over the $0.5-5 \mathrm{~nm}$ size range, with a maximum at around $1.5 \mathrm{~nm}$ (Figure 4c). The hydrodynamic diameter of UV-absorbing material at 575 $\mathrm{nm}$ (UV575) in the suboxic samples had its maximum at around $3 \mathrm{~nm}$. UV254 showed a secondary maximum, coinciding with the $3 \mathrm{~nm}$ maximum for samples $26 \mathrm{c}$ and $26 \mathrm{~d}$, this peak was not detected for sample 28d. In the aerated samples the UV575-signal was too low to be distinguished from the background, and the relative amount for UV254 in the aerated samples were less than half of those in the suboxic samples.

The material giving rise to a maximum UV254 at $1.5 \mathrm{~nm}$ is most likely fulvic or humic acid-like . ${ }^{[39]}$ This was also confirmed by excitation emission matrix fluorescence spectroscopy of bulk samples (Figure S2) and FIFFF coupled to fluorescence excitation emission spectroscopy specific to humic material (see Figure S3).

A comparison of number average adsorbed particle heights by AFM and number average hydrodynamic diameters by FIFFF are shown in Table 1 . In all cases average heights by AFM were larger by a factor of at least 5 compared to the number average values for the hydrodynamic diameters measured using FlFFF-UV absorbance. To some extent, the different size distributions achieved by AFM and FlFFF-UV can be explained by the AFM analysed particles being in a semi-dried state, while FlFFF-UV analysed them in liquid suspension. However, more important is probably the 
selectivity of FlFFF-UV for particles with strong UV-absorbance, such as humic and fulvic acids, which are typically found in the same 0.5-5 nm hydrodynamic diameter range as most of the UV254absorbing material detected by FIFFF-UV. Coupling other detectors to FIFFF, such as inductively coupled plasma mass spectrometry (ICP-MS), has shown that inorganic (e.g., iron-rich) colloids in natural waters are typically distributed towards larger hydrodynamic diameters relative to UVabsorbance ${ }^{[7,19,40]}$, closer to the average particle height determined by AFM in the present study. Nevertheless, it is surprising that such small fractions (usually around 10\%) of the particles detected by AFM were in the 0-5 nm hydrodynamic diameter range, where most UV254-absorbing matter was detected by FlFFF-UV, and where fulvic and humic acids are expected to be found. ${ }^{[39]}$ One mechanisms that could explain this result is the selective adhesion of near-neutrally charged particles (e.g., most iron oxyhydroxides at neutral $\mathrm{pH}$ ) over particles with strong negative charge (e.g., humic and fulvic acids) to the negatively charged mica ${ }^{[41]}$. It is possible extensive adhesion of humic and fulvic acids result in the formation of a surface film on the muscovite surface ${ }^{[42]}$ making it impossible to distinguish these particles in our samples using AFM. Formation of larger particles through the aggregation of humic and fulvic acids during AFM sample preparation is not likely to have occurred, because such aggregates are typically much more irregular in shape than the spherical particles detected in our samples by AFM ${ }^{[32]}$ and the method that we used for AFM sample preparation has been optimised to minimise such artefacts. ${ }^{[42]}$

Our findings illustrate that AFM and FlFFF-UV are complimentary techniques, detecting different types of NP in groundwater samples. The reduction of the UV254 peak at $1.5 \mathrm{~nm}$, and the removal of the UV575 peak at $3 \mathrm{~nm}$ in the FIFFF fractograms when the samples were aerated (Figures $4 \mathrm{~b}$ and $4 \mathrm{~d}$ ) could be explained by co-aggregation or incorporation of organic macromolecules in $\mathrm{Fe}$ or $\mathrm{Mn}$ rich colloids (see Figures S7 and S8 for elemental compositions), such as those observed by AFM as a result of aeration (Figure 2). In addition, the application of phase contrast AFM could also help distinguish between hard and soft colloids. ${ }^{[43]}$

Diffusion coefficients are important for understanding the transport of natural organic matter in the subsurface where movement within porewaters is dominated by molecular diffusion. Number average 
1 diffusion coefficients $\left(\mathrm{D}_{\mathrm{N}}\right)$ for organic NP calculated using the FlFFF-UV data (0-5 nm range) are

2 shown in Table 1. These were found to be between 3-3.3 $\times 10^{-10} \mathrm{~m}^{2} \mathrm{~s}^{-1}$ for suboxic samples and are $10-15 \%$ higher for aerated samples. These values are consistent with values obtained using model humic and fulvic (Suwannee River) substances $\left(2-3 \times 10^{-10} \mathrm{~m}^{2} \mathrm{~s}^{-1}\right)$ for comparable $\mathrm{pH}$ values using fluorescence correlation spectroscopy and FlFFF. ${ }^{[39]}$ The aeration experiments (Figure $4 \mathrm{~b}$ and $4 \mathrm{~d}$ ) show that there is contrasting reactivity between the two populations of organic NP, with the larger population (2-4 $\mathrm{nm}$ range) being rapidly removed while a proportion (ca. 30\%) of the smaller sized population (0.5-2 $\mathrm{nm}$ range) remained in solution. This could be explained in terms of higher reactivity to oxidation of higher molecular weight DOM fraction compared to lower molecular weight fractions due to size-charge ratio effects and greater repulsion of fulvic like DOM compared to humiclike DOM ${ }^{[44]}$ Understanding the temporal variability of DOM is important for understanding trace metal binding and transport in these dynamic floodplain environments as the lower molecular weight fulvic acid fraction is known to exhibit a much higher negative charge density, at a given $\mathrm{pH} /$ ionic strength, compared to humic acid fractions. ${ }^{[45]}$ The regular cycles of inundation may lead to rapid changes in the DOM characteristics as DOM is transported from the surface to depth within the alluvium and alluvial deposits.

Morphology and surface chemistry. A range of particles with differing sizes and morphology were observed from the TEM-micrographs of suboxic samples. Selected TEM micrographs from all four sites are shown in Figure 5 a-h illustrating the range of different NP the occurrence of few large $(>100$ $\mathrm{nm}$ ) colloids present in the suboxic groundwater. TEM images for the shallower groundwaters (Figure $5 \mathrm{~b}$ and $5 \mathrm{f}$ ) show that these samples are dominated by a large number of compact NP in the 5-20 nm size range, with both electron dense and poor regions. These NP dominate over larger colloids in both AFM and TEM samples. The regions which were more transparent to electrons are presumably rich in organics, while those that are dark are rich in Fe. These contrast with the results for the deeper sites (figure $5 \mathrm{~d}$ and $5 \mathrm{~h}$ ) which show a greater number of larger particles with more complex morphologies, corroborating the AFM results. Figure 5g illustrates the association of Fe rich (electron dense regions) and organic NP (electron poor regions), and the agglomeration of monomers to form macromolecules 
1 with complex shapes and high polydispersity, which again corroborates the results for the AFM 2 analysis (Table 1).

3 In the TEM micrographs, NP of a few nm in size were observed in the suboxic samples (Figure 5), but were not present in aerated samples (Figure S4, supplementary material). This is also consistent with the observations made using AFM. The presence of high dissolved and nanoparticulate Fe suggests that DOC from the landfill has mobilised Fe oxides by reductive dissolution and produced in-situ colloidal and nanoparticulate Fe oxides. ${ }^{[30]}$ Fibrillar material was also observed in association with the colloidal sized material in sample 28c (Figure 5e), presumably indicating a source of biopolymeric organic matter in the shallow groundwater. The fact that the larger colloids $(>50 \mathrm{~nm})$ were observed in the suboxic samples by TEM but not by AFM can be explained by the fact that the probability of finding them with AFM was much lower, since AFM-images were acquired on random locations of the mica substrate, while TEM-micrographs were acquired on particles selected after searching large areas of the TEM-grids. In addition, the electron dense particles show up clearly on the TEM images while less dense (e.g. organic) are less obvious. The formation of NP by the oxidation of iron and manganese by the electron beam cannot be ruled out. However, such perturbations are assumed to be minimal, since no changes of the samples could be observed during TEM-analysis.

SEM micrographs taken for suboxic samples from all four sites are given in Figures S5-S8. These showed colloids with similar shapes as the larger electron dense colloids on the TEM-micrographs. EDX (energy dispersive X-ray spectroscopy) enabled the determination of major element composition at selected locations. The EDX results show two different types of colloids that dominated these samples: i) larger colloids rich in $\mathrm{Ca}$ and $\mathrm{O}$ (as calcite, given these samples are calcite saturated) with minor amounts of $\mathrm{Fe}, \mathrm{Mn}, \mathrm{S}, \mathrm{Al}$ and $\mathrm{Si}$, ii) $\mathrm{Fe}, \mathrm{Mn}$ and oxygen rich colloids (Fe/Mn-oxyhydroxides) associated with more minor quantities of $\mathrm{Ca}$ and $\mathrm{P}$.

\section{Acknowledgments}

Analytical work at the Natural Environment Research Councils (NERC) Facility for Environmental Nanoparticle Analysis and Characterisation (FENAC) was funded under project 
1 FENAC/2009/12/001. Kate Griffiths and David Macdonald (BGS) are thanked for supervising the

2 instrumentation of the Oxford site. Andy Dixon (Groundwater Monitoring and Drilling Ltd) is

3 thanked for carrying out installation of piezometers. Oxford City Council is thanked for giving

4 permission to access the field site. This manuscript is published with the permission of the BGS

5 director.

6 Supporting Information Available

7 Text, tables and figures describing the study site, groundwater chemistry, FlFFF-fluorescence results,

8 TEM/SEM micrographs of aerated samples and summary EDX results. This information is available

9 free of charge via the Internet at http://pubs.acs.org/.

11 Tables: 
1 Table 1. Summary table of number and weight average particle heights by AFM, polydispersity

2 index, number average hydrodynamic diameter and diffusion coefficients for organic NP by

3 FIFFF-UV

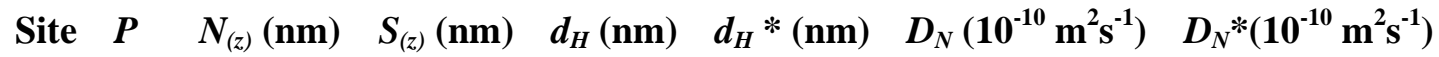

\begin{tabular}{llllllll}
\hline 26c & 1.2 & 11.7 & 13.5 & 2.0 & 1.4 & $3.04 \mathrm{E}-10$ & $3.51 \mathrm{E}-10$ \\
$26 \mathrm{~d}$ & 1.4 & 11.3 & 15.7 & 1.8 & 1.3 & $3.2 \mathrm{E}-10$ & $3.45 \mathrm{E}-10$ \\
$28 \mathrm{c}$ & 1.2 & 10.0 & 11.7 & N/A & N/A & N/A & N/A \\
$28 \mathrm{~d}$ & 2.1 & 9.2 & 19.5 & 1.6 & N/A & $3.29 \mathrm{E}-10$ & N/A
\end{tabular}

$4 \overline{N_{(z)}}$ is the number average particle height by AFM, $S_{(z)}$ is the weight average particle height by AFM. $D_{N}$ is the number average diffusion

5 coefficient by FIFFF-UV ( $245 \mathrm{~nm}$ for the $0-10 \mathrm{~nm}$ range), $d_{H}$ is the number average hydrodynamic diameter by FIFFF-UV ( $245 \mathrm{~nm}$ for the

$6 \quad 0-10 \mathrm{~nm}$ range), * indicates values for aerated samples

7

8

9

10

11 


\section{Sub-oxic sampling}

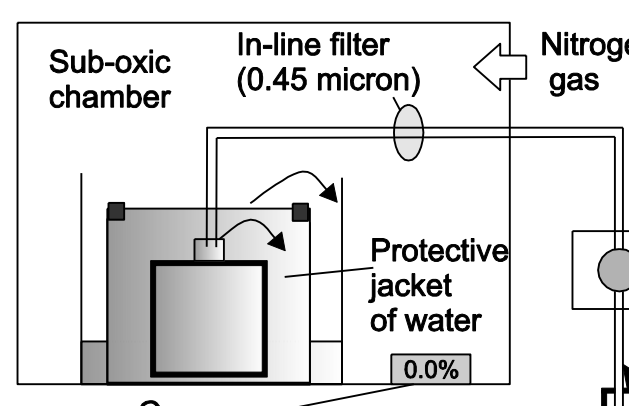

3

4 5
$\Rightarrow \quad$ Storage

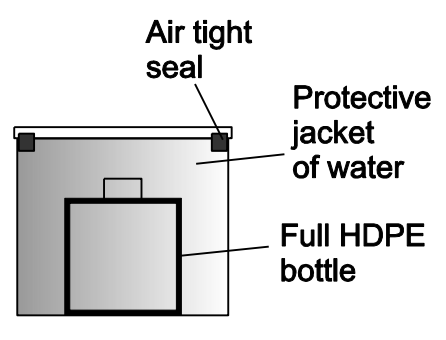

Peristaltic pump Oxygen probe

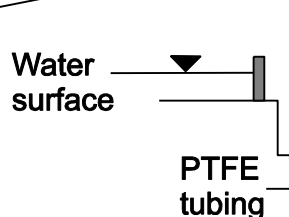

Extendable tube for sampling during inundation tubing - Water tight bund Ground surface

Figure 1. Schematic of suboxic groundwater sampling during inundation and storage methodology. 


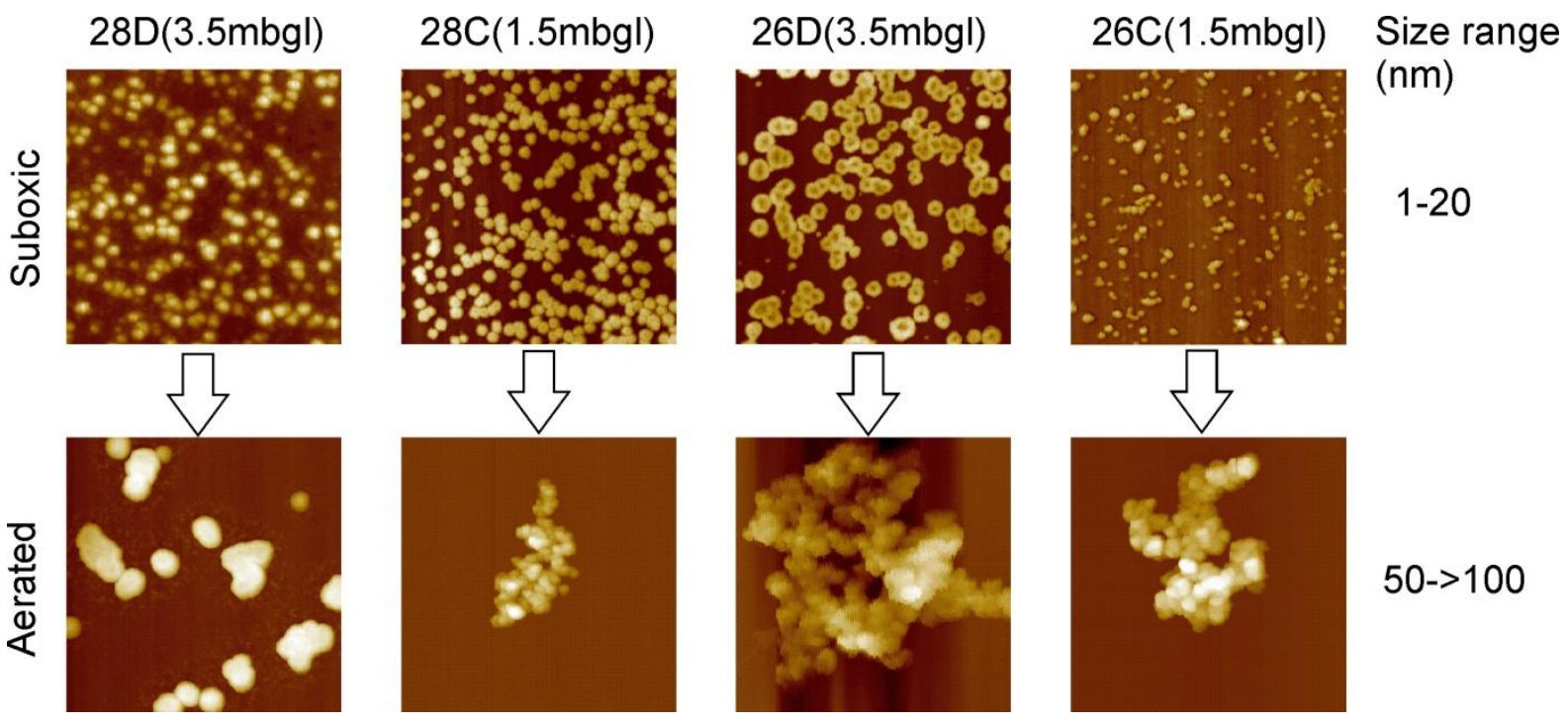

2 Figure 2. Typical AFM scans $(1 \times 1 \mu \mathrm{m})$ for all four samples before and after aeration experiments.

3 Aeration carried out by bubbling with compressed air for 10 minutes. 

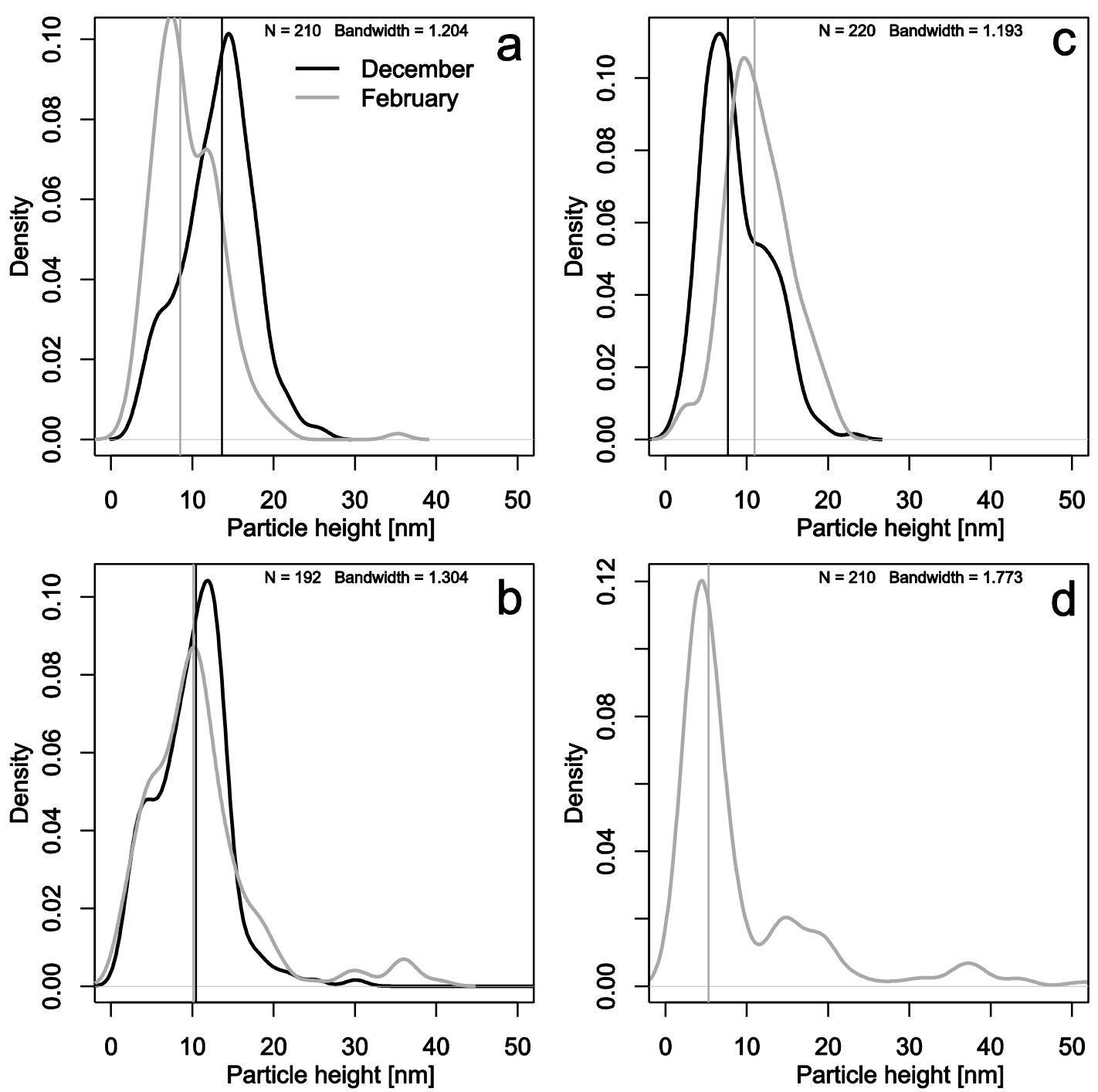

2 Figure 3. Kernel density estimates for suboxic AFM results for all four sites: (a) 26c, (b) 26d, (c) 28c and (d) 28d. Results from December 2010 sampling round shown in black, results from February round are shown in grey. Results comprise $>190$ individual measurements $(\mathrm{N})$ for each sample, AFM

5 carried out in a $\mathrm{N}_{2}$ atmosphere following absorption to mica under suboxic conditions. Vertical lines

6 show median values for the distributions. Kernel density estimates ${ }^{[46]}$ are similar to histograms but

7 represent a continuous, smooth, distribution giving a more realistic representation of the particle

8 height distributions, removing the dependence on the end points of bins and minimising bin width

9 effects. A Gaussian kernel was used and the smoothing bandwidth was optimised and scaled to be the standard deviation of the smoothing kernel. 

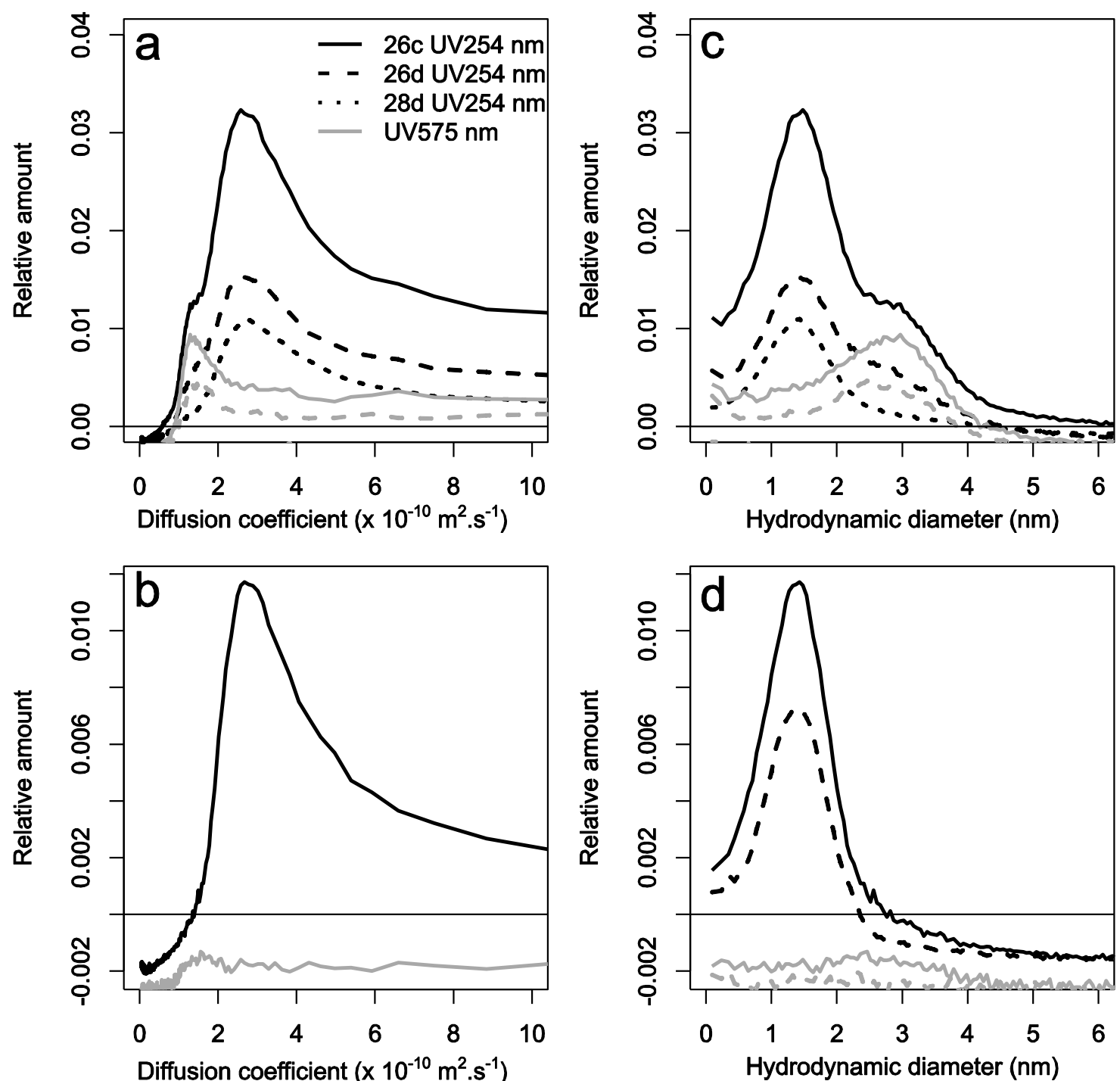

2 Figure 4. FlFFF fractograms: (a) diffusion coefficient for suboxic samples, (b) diffusion coefficient 3 for aerated samples, (c) hydrodynamic diameter for suboxic samples, (d) hydrodynamic diameter for 4 aerated samples. Plots in black show result for UV 254 signal, plots in grey show results for UV 575 $5 \mathrm{~nm}$. FlFFF setup: channel flow of $0.5 \mathrm{~mL} \mathrm{~min}^{-1}$ and a focus flow of $2.5 \mathrm{~mL} \mathrm{~min}^{-1}$ for $30 \mathrm{~min}$, elution 6 and fractionation carried out with a crossflow of $3 \mathrm{~mL} \mathrm{~min}{ }^{-1}$ and detector flow of $0.5 \mathrm{~mL} \mathrm{~min}{ }^{-1}$.

7 Suboxic analysis carried out after degassing buffer solution with $\mathrm{N}_{2}$. 


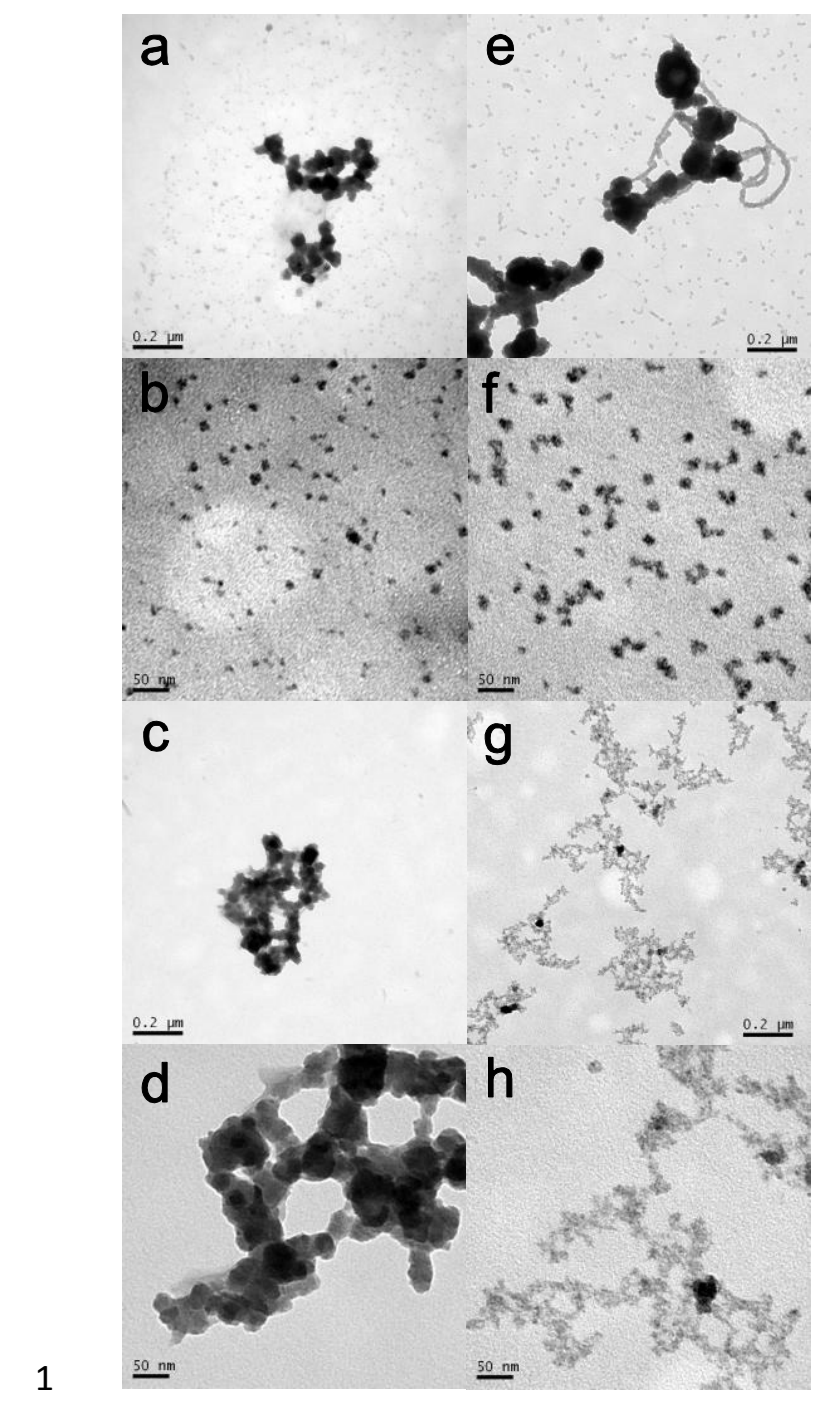

2 Figure 5. Typical TEM micrographs for suboxic samples: (a) 26c, (b) 26c, (c) 26d, (d) 26d, (e) 28c, 3 (f) $28 \mathrm{c},(\mathrm{g}) 28 \mathrm{~d}$, (h) $28 \mathrm{~d}$. 
1. Grolimund, D.; Borkovec, M.; Barmettler, K.; Sticher, H., Colloid-facilitated transport of strongly sorbing contaminants in natural porous media: A laboratory column study. Environ Sci Technol 1996, 30, (10), 3118-3123.

2. $\quad$ Degueldre, C.; Triay, I.; Kim, J. I.; Vilks, P.; Laaksoharju, M.; Miekeley, N., Groundwater colloid properties: a global approach. Appl Geochem 2000, 15, (7), 1043-1051.

3. Lead, J. R.; Wilkinson, K. J., Aquatic colloids and nanoparticles: Current knowledge and future trends. Environ Chem 2006, 3, (3), 159-171.

4. Waychunas, G. A.; Kim, C. S.; Banfield, J. F., Nanoparticulate iron oxide minerals in soils and sediments: unique properties and contaminant scavenging mechanisms. J Nanopart Res 2005, 7, (4-5), 409-433.

5. $\quad$ Speelmans, M.; Vanthuyne, D. R. J.; Lock, K.; Hendrickx, F.; Du, L. G.; Tack, F. M. G.; Janssen, C. R., Influence of flooding, salinity and inundation time on the bioavailability of metals in wetlands. Sci Total Environ 2007, 380, (1-3), 144-153.

6. Jensen, D. L.; Ledin, A.; Christensen, T. H., Speciation of heavy metals in landfill-leachate polluted groundwater. Water Res 1999, 33, (11), 2642-2650.

7. $\quad$ Kersting, A. B.; Efurd, D. W.; Finnegan, D. L.; Rokop, D. J.; Smith, D. K.; Thompson, J. L., Migration of plutonium in ground water at the Nevada Test Site. Nature 1999, 397, (6714), 56-59.

8. Dai, M.; Kelley, J. M.; Buesseler, K. O., Sources and migration of plutonium in groundwater at the Savannah River Site. Environ Sci Technol 2002, 36, (17), 3690-3699.

9. Hassellöv, M.; Buesseler, K. O.; Pike, S. M.; Dai, M., Application of cross-flow ultrafiltration for the determination of colloidal abundances in suboxic ferrous-rich ground waters. Sci Total Environ 2007, 372, (2-3), 636-644.

10. Bargar, J. R.; Bernier-Latmani, R.; Giammar, D. E.; Tebo, B. M., Biogenic Uraninite Nanoparticles and Their Importance for Uranium Remediation. Elements 2008, 4, (6), 407-412.

11. Utsunomiya, S.; Kersting, A. B.; Ewing, R. C., Groundwater Nanoparticles in the Far-Field at the Nevada Test Site: Mechanism for Radionuclide Transport. Environ Sci Technol 2009, 43, (5), 1293-1298.

12. Dai, M.; Martin, J. M.; Cauwet, G., The Significant Role of Colloids in the Transport and Transformation of Organic-Carbon and Associated Trace-Metals (Cd, $\mathrm{Cu}$ and $\mathrm{Ni})$ in the Rhone Delta (France). Mar Chem 1995, 51, (2), 159-175.

13. Noell, A. L.; Thompson, J. L.; Corapcioglu, M. Y.; Triay, I. R., The role of silica colloids on facilitated cesium transport through glass bead columns and modeling. J Contam Hydrol 1998, 31, (12), 23-56.

14. Baalousha, M.; Kammer, F. V. D.; Motelica-Heino, M.; Baborowski, M.; Hofmeister, C.; Le Coustumer, P., Size-based speciation of natural colloidal particles by flow field flow fractionation, inductively coupled plasma-mass spectroscopy, and transmission electron microscopy/X-ray energy dispersive spectroscopy: Colloids-trace element interaction. Environ Sci Technol 2006a, 40, (7), 2156-2162.

15. Balnois, E.; Wilkinson, K. J.; Jr, L.; Buffle, J., Atomic force microscopy of humic substances: Effects of pH and ionic strength. Environ Sci Technol 1999, 33, (21), 3911-3917.

16. Lead, J. R.; Muirhead, D.; Gibson, C. T., Characterization of freshwater natural aquatic colloids by atomic force microscopy (AFM). Environ Sci Technol 2005, 39, (18), 6930-6936.

17. Redwood, P. S.; Lead, J. R.; Harrison, R. M.; Jones, I. P.; Stoll, S., Characterization of humic substances by environmental scanning electron microscopy. Environ Sci Technol 2005, 39, (7), $1962-$ 1966.

18. Baalousha, M.; Lead, J. R., Characterization of natural aquatic colloids $(<5 \mathrm{~nm})$ by flow-field flow fractionation and atomic force microscopy. Environ Sci Technol 2007, 41, (4), 1111-1117. 
19. Stolpe, B.; Hassellov, M.; Andersson, K.; Turner, D. R., High resolution ICPMS as an on-line detector for flow field-flow fractionation; multi-element determination of colloidal size distributions in a natural water sample. Anal Chim Acta 2005, 535, (1-2), 109-121.

20. Wyatt, T.; Jenkinson, I.; Malej, A., How viscoelastic properties of colloids, transparent exopolymeric particles and marine organic aggregates, modify turbulence and plankton biodynamics in the sea. Progress and Trends in Rheology V 1998, 65-66.

21. Contado, C.; Blo, G.; Fagioli, F.; Dondi, F.; Beckett, R., Characterisation of River Po particles by sedimentation field-flow fractionation coupled to GFAAS and ICP-MS. Colloid Surface A 1997, 120, (1-3), 47-59.

22. Baalousha, M.; Kammer, F. V. D.; Motelica-Heino, M.; Hilal, H. S.; Le Coustumer, P., Size fractionation and characterization of natural colloids by flow-field flow fractionation coupled to multi-angle laser light scattering. J Chromatogr A 2006b, 1104, (1-2), 272-281.

23. Hassellov, M.; Lyven, B.; Haraldsson, C.; Sirinawin, W., Determination of continuous size and trace element distribution of colloidal material in natural water by on-line coupling of flow fieldflow fractionation with ICPMS. Anal Chem 1999, 71, (16), 3497-3502.

24. Wolthoorn, A.; Temminghoff, E. J. M.; van Riemsdijk, W. H., Colloid formation in groundwater by subsurface aeration: characterisation of the geo-colloids and their counterparts. Appl Geochem 2004, 19, (9), 1391-1402.

25. Wolthoorn, A.; Temminghoff, E. J. M.; Weng, L. P.; van Riemsdijk, W. H., Colloid formation in groundwater: effect of phosphate, manganese, silicate and dissolved organic matter on the dynamic heterogeneous oxidation of ferrous iron. Appl Geochem 2004, 19, (4), 611-622.

26. Backhus, D. A.; Ryan, J. N.; Groher, D. M.; Macfarlane, J. K.; Gschwend, P. M., Sampling Colloids and Colloid-Associated Contaminants in Ground-Water. Ground Water 1993, 31, (3), 466479 .

27. Oster, H.; Sonntag, C.; Munnich, K. O., Groundwater age dating with chlorofluorocarbons. Water Resour Res 1996, 32, (10), 2989-3001.

28. Mccave, I. N., Size Spectra and Aggregation of Suspended Particles in the Deep Ocean. Deep-Sea Res 1984, 31, (4), 329-352.

29. Handy, R. D.; von der Kammer, F.; Lead, J. R.; Hassellov, M.; Owen, R.; Crane, M., The ecotoxicology and chemistry of manufactured nanoparticles. Ecotoxicology 2008, 17, (4), 287-314.

30. Liang, L. Y.; Mccarthy, J. F.; Jolley, L. W.; Mcnabb, J. A.; Mehlhorn, T. L., Iron Dynamics Transformation of $\mathrm{Fe}(\mathrm{Ii}) / \mathrm{Fe}$ (Iii) during Injection of Natural Organic-Matter in a Sandy Aquifer. Geochim Cosmochim Ac 1993, 57, (9), 1987-1999.

31. Dondi, F.; Martin, M., In Field-Flow Fractionaction Handbook, Wiley-Interscience: New York, 2000; p 103.

32. Wilkinson, K. J.; Balnois, E.; Leppard, G. G.; Buffle, J., Characteristic features of the major components of freshwater colloidal organic matter revealed by transmission electron and atomic force microscopy. Colloid Surface A 1999, 155, (2-3), 287-310.

33. Shapiro, S. S.; Wilk, M. B., An analysis of variance test for normality (complete samples). Biometrika 1965, 52, 591-611.

34. Breslow, N., Generalized Kruskal-Wallis Test for Comparing K Samples Subject to Unequal Patterns of Censorship. Biometrika 1970, 57, (3), 579-\&.

35. Siegel, S., Nonparametric statistics for the behavioral science. McGraw-Hill: 1956; p 312.

36. Holocher, J.; Matta, V.; Aeschbach-Hertig, W.; Beyerle, U.; Hofer, M.; Peeters, F.; Kipfer, R., Noble gas and major element constraints on the water dynamics in an alpine floodplain. Ground Water 2001, 39, (6), 841-852.

37. MacDonald, D. M. J.; Griffiths, K. J.; Lapworth, D. J.; Williams, P. J.; Stuart, M. E.; Gooddy, D. C., Hydrogeochemical characterisation of a peri-urban floodplain: initial findings. In Report, B. G. S. I., Ed. 2012; p 97.

38. Buffle, J.; Wilkinson, K. J.; Stoll, S.; Filella, M.; Zhang, J. W., A generalized description of aquatic colloidal interactions: The three-colloidal component approach. Environ Sci Technol 1998, 32, (19), 2887-2899.

39. Jr, L.; Wilkinson, K. J.; Balnois, E.; Cutak, B. J.; Larive, C. K.; Assemi, S.; Beckett, R., Diffusion coefficients and polydispersities of the Suwannee River fulvic acid: Comparison of 
1 fluorescence correlation spectroscopy, pulsed-field gradient nuclear magnetic resonance, and flow field-flow fractionation. Environ Sci Technol 2000, 34, (16), 3508-3513.

40. Hassellov, M.; von der Kammer, F., Iron Oxides as Geochemical Nanovectors for Metal Transport in Soil-River Systems. Elements 2008, 4, (6), 401-406.

41. Baalousha, M., Aggregation and disaggregation of iron oxide nanoparticles: Influence of particle concentration, pH and natural organic matter. Sci Total Environ 2009, 407, (6), 2093-2101.

42. Balnois, E.; Wilkinson, K. J., Sample preparation techniques for the observation of environmental biopolymers by atomic force microscopy. Colloid Surface A 2002, 207, (1-3), 229-242. 43. Plaschke, M.; Romer, J.; Kim, J. I., Characterization of Gorleben groundwater colloids by atomic force microscopy. Environ Sci Technol 2002, 36, (21), 4483-4488.

44. Christl, I.; Milne, C. J.; Kinniburgh, D. G.; Kretzschmar, R., Relating ion binding by fulvic and humic acids to chemical composition and molecular size. 2. Metal binding (vol 35, pg 2512, 2001). Environ Sci Technol 2001, 35, (13), 2860-2860.

45. Christl, I.; Kretzschmar, R., Relating ion binding by fulvic and humic acids to chemical composition and molecular size. 1. Proton binding (vol 35, pg 2505, 2001). Environ Sci Technol 2001, $35,(13), 2860-2860$.

46. Jones, M. C.; Marron, J. S.; Sheather, S. J., A brief survey of bandwidth selection for density estimation. J Am Stat Assoc 1996, 91, (433), 401-407. 